\title{
PASCAL TYPE PROPERTIES OF BETTI NUMBERS
}

\section{TILAK DE ALWIS}

Department of Mathematics

Southeastern Louisiana University

Hammond, Louisiana 70402

(Received January 13, 1993)

\begin{abstract}
In this paper, we will describe the Pascal Type properties of Betti numbers of ideals associated to $n$-gons. These are quite similar to the properties enjoyed by the Pascal's Triangle, concerning the binomial coefficients. By definition, the Betti numbers $\beta_{1}(n)$ of an ideal $I$ associated to an $n$-gon are the ranks of the modules in a free minimal resolution of the $R$ module $R / I$, where $R$ is the polynomial ring $k\left[x_{1}, x_{2}, \cdots, x_{n}\right]$. Here $k$ is any field and $x_{1}, x_{2}, \cdots, x_{n}$ are indeterminates. We will prove those properties using a specific formula for the Betti numbers.
\end{abstract}

KEY WORDS AND PHRASES. Betti numbers, finite abstract simplicial complex, StanleyReisner ideal, free minimal resolutions, Koszul complex, double complex.

1991 AMS SUBJECT CLASSIFICATION CODE. $13 \mathrm{H} 10$.

\section{INTRODUCTION.}

In this paper, we will describe the so-called Pascal Type properties of the Betti numbers of ideals associated to $n$-gons.

In order to explain what these Betti numbers are, consider the general $n$-gon $(n \geq 3)$ with vertices at the points $1,2, \cdots, n$ (say, labeled anticlockwise). This corresponds to a finite abstract simplicial complex,

$$
\Delta=\{\emptyset,\{1\},\{2\}, \cdots,\{n\},\{1,2\},\{2,3\}, \cdots,\{n-1, n\},\{1, n\}\} .
$$

In other words, $\Delta$ consists of vertex sets and edge sets of the $n$-gon together with the empty set. For the general definition of a finite abstract simplicial complex, the reader may refer to [1], [2] or [3].

Let $R$ be the polynomial ring $k\left[x_{1}, x_{2}, \cdots, x_{n}\right]$ where $k$ is any field and $x_{i}$ are indeterminates. Let $I$ be the ideal in $R$ generated by all the monomials of the form $x_{i_{1}} x_{i_{2}} \cdots x_{i_{r}}$ with $1 \leq i_{1}<i_{2}<\cdots<i_{r} \leq n$ and $\left\{i_{1}, i_{2}, \cdots, i_{r}\right\} \notin \Delta$.

The ring $R / I$ is known as the Stanley-Reisner ring or the face ring of the finite abstract simplicial complex $\Delta$ (See [3]). The ideal $I$ is also known as the Stanley-Reisner ideal.

A free minimal resolution of the $R$-module $R / I$ is an exact sequence of the form

$$
\cdots \rightarrow M_{2} \rightarrow \cdots \rightarrow M_{1} \rightarrow M_{0} \rightarrow R / I \rightarrow 0
$$

where each $M_{1}$ is a free $R$-module with the smallest possible rank. For material on free minimal resolutions, the reader can refer to [3] or [4].

Then the Betti numbers $\beta_{\mathfrak{z}}(n)$ of the $R$-module $R / I$ are just the ranks of those free modules $M_{\text {v }}$,i.e., $\quad \beta_{\imath}(n)=\operatorname{rank}_{R}\left(M_{\mathrm{q}}\right) \quad$ for $\quad i=0,1,2, \cdots \quad$ It can also be shown that $\beta_{\imath}(n)=\operatorname{dim}_{k}\left[\operatorname{Tor}_{i}^{R}(R / I, k)\right]$. By abuse of language, we will also refer to them as the Betti 
numbers of the ideal $I$, or simply the Betti numbers of the $n$-gon.

Before establishing the Pascal Type properties of Betti numbers $\beta_{1}(n)$, we will first describe how to obtain a formula for $\beta_{\imath}(n)$. The next section achieves this.

\section{THE BETTI NUMBERS OF THE $\boldsymbol{n}$-GON.}

THEOREM 2.1. Let $I_{j}=\left(x_{1}, x_{2}, \cdots, \widehat{x}_{j}, \widehat{x}_{j+1}, \cdots, x_{n}\right) j=1,2, \cdots, n$ where - denotes the omission. Then

$$
0 \rightarrow \frac{R}{I} \stackrel{\rho}{\rightarrow} \stackrel{n}{\oplus}=1 \frac{R}{I_{j}} \stackrel{g}{\rightarrow} \stackrel{n}{\stackrel{\oplus}{=}} \frac{R}{I_{j}+I_{3}+1} \stackrel{h}{\rightarrow} \frac{R}{I_{1}+I_{2}+\cdots+I_{n}} \rightarrow 0
$$

is a co-resolution of $R / I$. Here,

$$
\begin{gathered}
f(\bar{p})=(\bar{p}, \bar{p}, \cdots, \bar{p}) \\
g\left(\overline{q_{1}}, \overline{q_{2}}, \cdots, \overline{q_{n}}\right)=\left(\overline{-q_{1}+q_{2}}, \overline{-q_{2}+q_{3}}, \cdots, \overline{-q_{n-1}+q_{n}}, \overline{-q_{n}+q_{1}}\right) \\
h\left(r_{1}, r_{2}, \cdots, r_{n}\right)=\left(\overline{r_{1}+r_{2}+\cdots+r_{n}}\right)
\end{gathered}
$$

In the above, when $j=n, I_{3+1}$ is read as $I_{1}$.

PROOF. For the complete proof, see [5].

In other words, the above result means that the following complex is exact at all the places except at the 0 th spot, at which it has homology equal to $R / I$.

$$
0 \rightarrow \underset{j=1}{\oplus} \frac{R}{I_{j}} \stackrel{g}{\rightarrow} \underset{j=1}{\stackrel{n}{=}} \frac{-1}{I_{j}+I_{\jmath+1}} \stackrel{h}{\rightarrow} \frac{R}{I_{1}+I_{2}+\cdots+I_{n}} \rightarrow 0
$$

Notice that for any $j$, each of $I_{\jmath}, I_{\jmath}+I_{\jmath+1}$ and $I_{1}+I_{2}+\cdots+I_{n}$ is generated by a regular sequence of length $n-2, n-1$ and $n$ respectively. Hence, we can use Koszul complex resolutions to lift the complex $(2.1)$ into a double complex $\left(C_{i,}, \delta_{i,}, d_{i, j}\right)$ as given in our next Theorem 2.4. For material on regular sequences and Koszul complexes, the reader can refer to [1], [3] or [4]. Let us first give some definitions.

DEFINITION 2.2. A nonempty subset $S=\left\{r_{1}, r_{2}, \cdots, r_{j}\right\}$ of $\{1,2, \cdots, n\}$ is said to have consecutive elements if there is a permutation $\rho$ of $\left\{r_{1}, r_{2}, \cdots, r_{3}\right\}$ such that $\rho\left(r_{t+1}\right)=\rho\left(r_{t}\right)+1,1 \leq t \leq j-1$. Here, $\rho\left(r_{t}\right)+1$ is read as 1 , whenever $\rho\left(r_{t}\right)=n$.

\section{DEFINITION 2.3.}

(a) For $i=0,-1$ and arbitrary $j$, let $C_{i,}$, be the free $R$-module having basis as the set, $\{(U, S)|S \subseteq\{1,2, \cdots, n\}| S \mid,=1-i, S$ has consecutive elements, $U \subseteq U(S),|U|=j\}$ and,

(b) For $i=-2$ and arbitrary $j$, let $C_{i}$, be the free $R$-module having basis as the set $\{U, S)|S \subseteq\{1,2, \cdots, n\}| S,|=n, U \subseteq U(S)| U \mid,=j\}$, which is the same as $\{(U,\{1,2, \cdots, n\})|U \subseteq U(\{1,2, \cdots, n\})| U \mid,=j\}$.

In the above definitions for $C_{i, j}$, for $S \subseteq\{1,2, \cdots, n\}$, the notation $U(S)$ just means $\bigcup_{\in} U_{3}$, where $U$, is the set of indeterminates used to define the ideal $I$, (see [6], page 2).

One can also observe that,

$$
\begin{aligned}
& \operatorname{rank}_{R}\left(C_{0, \jmath}\right)=\left\{\begin{array}{cl}
n\left(\begin{array}{c}
n-2 \\
j
\end{array}\right) & \text { for } j=0,1, \cdots, n-2 \\
0 & \text { for } j=n-1, n
\end{array}\right. \\
& \operatorname{rank}_{R}\left(C_{-1, j}\right)=\left\{\begin{array}{cl}
n\left(\begin{array}{c}
n-1 \\
j
\end{array}\right) & \text { for } j=0,1, \cdots, n-1 \\
0 & \text { for } j=n
\end{array}\right. \\
& \operatorname{rank}_{R}\left(C_{-2, \jmath}\right)=\left(\begin{array}{c}
n \\
j
\end{array}\right) \quad \text { for } j=0,1, \cdots, n
\end{aligned}
$$


Also, define the maps

$$
\begin{aligned}
& \alpha: C_{0,0} \rightarrow \stackrel{n}{\oplus} \stackrel{n}{=} \frac{R}{I_{j}}, \quad \beta: C_{-1,0} \rightarrow \stackrel{n}{\oplus} \frac{R}{I_{1}+I_{\jmath+1}} \\
& \gamma: C_{-2,0} \rightarrow \frac{R}{I_{1}+I_{2}+\cdots+I_{n}} \\
& d_{1, j}: C_{1, j} \rightarrow C_{1, j-1}(\imath=0,-1,-2 \text { and } j=1,2, \cdots, n) \\
& \delta_{1, j}: C_{1, j} \rightarrow C_{1-1, j}(i=0,-1 \text { and } j=0,1, \cdots, n)
\end{aligned}
$$

respectively by,

$$
\begin{aligned}
& \alpha(\emptyset,\{r\})=\overline{(0, \cdots, 1, \cdots, 0)}, \quad \beta(\emptyset,\{r, r+1\})=\overline{(0, \cdots, 1, \cdots, 0)} \\
& \gamma(\emptyset,\{1,2, \cdots, n\})=\overline{1} \\
& d_{i, j}\left(\left\{x_{p_{1}}, \cdots, x_{p_{j}}\right\}, S\right)=\sum_{k=1}^{j}(-1)^{k-1} x_{p_{k}}\left(\left\{x_{p_{1}}, \cdots, \widehat{x}_{p_{k}}, \cdots, x_{p_{\jmath}}\right\}, S\right) \\
& \delta_{0, j}(U,\{r\})=(U,\{r-1, r\})-(U,\{r, r+1\}) \\
& \delta_{-1, j}(U,\{r, r+1\})=(U,\{1,2, \cdots, n\})
\end{aligned}
$$

Then we have the following theorem.

THEOREM 2.4. The following is a double complex

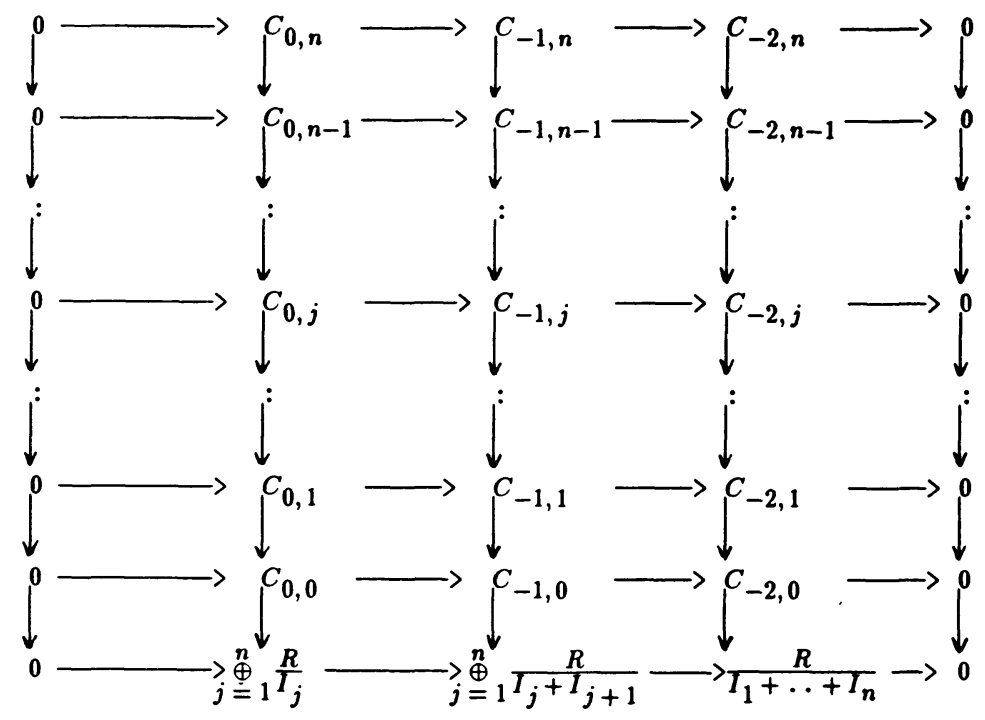

PROOF. The proof is a very direct calculation.

We will denote the above double complex by $\left(C_{i, j}, \delta_{i, j}, d_{i, j}\right)$. Before proving some properties of the horizontal maps $\delta_{i}$, of this double complex, we need some preparatory work.

First, introduce some notation. For a given $U \subseteq\left\{x_{1}, x_{2}, \cdots, x_{n}\right\}$ with $|U|=j$, we will define three $R$-modules by,

$$
\begin{aligned}
& D_{0}(U)=\underset{\substack{1 \leq r \leq n \\
U \subseteq U(\{r\})}}{\oplus}\langle(U,\{r\})\rangle, \\
& D_{-1}(U)=\underset{\substack{1 \leq r \leq n \\
U \subseteq U(\{r-1, r\})}}{\oplus}\langle(U,\{r-1, r\})\rangle, \\
& D_{-2}(U)=\langle(U,\{1,2, \cdots, n\})\rangle
\end{aligned}
$$

In the above, $\langle(U,\{r\})\rangle$ means the $R$-submodule of $C_{0}$, generated by the basis element $(U,\{r\})$, etc. 
THEOREM 2.5. Fix $U \subseteq\left\{x_{1}, x_{2}, \cdots, x_{n}\right\}$ with $|U|=\jmath$. Then we have,

(a) $\quad C_{\imath, \jmath}=\underset{|U|=\jmath}{\bigoplus} D_{\imath}(U)$ for $\imath=0,-1,-2$.

(b) $\left(D .(U), \delta_{. j}(U)\right)$ is a sub chain complex of $C_{. j}$ where $\delta_{. j}(U)$ denotes the map $\delta_{.,}$restricted to $D_{.(U)}$.

PROOF. Easily follows from the definitions of $C_{i,}, D_{\mathfrak{i}}(U)$ and $\delta_{\mathrm{i},}$.

Now, for $U \subseteq\left\{x_{1}, x_{2}, \cdots, x_{n}\right\}$ define three sets as follows. $V(U)=\left\{\{r\} \mid x_{r} \in U\right\}, E(U)$ $=\left\{\{r-1, r\} \mid x_{r} \in U\right\} \cup\left\{\{r, r+1\} \mid x_{r} \in U\right\}$ and $\Gamma(U)=\Delta-(V(U) \cup E(U))$, where $\Delta=\{\emptyset,\{1\},\{2\}$, $\cdots,\{n\},\{1,2\},\{2,3\}, \cdots,\{n-1, n\}\{1, n\}\}$ was defined at the very beginning.

THEOREM 2.6. For any $U \subseteq\left\{x_{1}, x_{2}, \cdots x_{n}\right\}, \Gamma(U)$ is a finite abstract simplicial complex, which is a subcomplex of $\Delta$.

PROOF. It is essentially showing that $\{r, r+1\} \in \Gamma(U) \Rightarrow\{r\} \in \Gamma(U)$ and $\{r+1\} \in \Gamma(U)$. But this follows readily from above definitions of $V(U), E(U)$ and $\Gamma(U)$.

Now, let $(\Gamma .(U), \gamma .(U))$ be the reduced chain complex corresponding to $\Gamma(U)$, namely the nonzero modules $\Gamma .(U)$ and the differential maps $\gamma .(U)$ between them are defined as follows.

$\Gamma_{1}(U)=R$-module generated by the edges $\{r-1, r\}$ of $\Gamma(U)$

$\Gamma_{0}(U)=R$-module generated by the vertices $\{r\}$ of $\Gamma(U)$

$\Gamma_{-1}(U)=R$

and, $\gamma_{1}(U)=\{r-1, r\}-\{r\}, \gamma_{0}(U)=1$, the identity element of $R$. Also, let $\left(\Lambda .(U), \lambda_{.}(U)\right)$ be the chain complex obtained by shifting the complex $(\Gamma .(U), \gamma .(U))$ one degree to the right. That is $\Lambda_{\mathrm{s}}(U)=\Gamma_{\mathrm{i}+1}(U)$ and $\lambda_{\mathrm{t}}(U)=\gamma_{\mathrm{t}+1}(U)$ for $i=0,-1,-2$.

THEOREM 2.7. For any $U \subseteq\left\{x_{1}, x_{2}, \cdots, x_{n}\right\}$, the chain complex $\left(\Lambda_{.}(U), \lambda_{.}(U)\right)$ is isomorphic to the chain complex $\left(D .(U), \delta_{. j}(U)\right)$.

PROOF. Producing an isomorphism $\phi$. from $\Lambda_{.}(U)$ to $D .(U)$ will establish the theorem. So, define $\phi$. on the basis elements as $\phi_{0}(\{r-1, r\})=(U,\{r-1\}), \phi_{-1}(\{r\})=(U,\{r-1, r\})$ and $\phi_{-2}(1)=(U,\{1,2, \cdots, n\})$, and then extend linearly. Then it is easy to check that all the squares commute, i.e., $\phi_{-2} \circ \lambda_{-1}(U)=\delta_{-1,},(U) \circ \phi_{-1}$ and $\phi_{-1} \circ \lambda_{0}(U)=\delta_{0,},(U) \circ \phi_{0}$. Hence the theorem.

THEOREM 2.8. In the double complex $\left(C_{i, j}, \delta_{i, j}, d_{i, j}\right)$ given in Theorem 2.4,

(a) The maps $\delta_{0}$, are injective for $j=1,2, \cdots, n$.

(b) The maps $\delta_{-1,}$, are surjective for $j=0,1, \cdots, n-1$.

PROOF.

(a) Let $\emptyset \neq U \subseteq\left\{x_{1}, x_{2}, \cdots, x_{n}\right\}$. Notice that $\emptyset \neq U$ means the same as $j \neq 0$. Hence, if $j=n-1$, or $n$, by definition $D_{0}(U)$ is zero and $\delta_{0,},(U)$ is injective for those values of $j$. On the other hand, for the other values of $j=1,2, \cdots, n-2, \Gamma(U)$ corresponds to a finite union of disjoint lines and points. Therefore by Theorem 2.7, ker $\delta_{0,},(U)=H_{0}(\Lambda .(U))=H_{1}(\Gamma .(U))$ which is zero by elementary topology. Hence $\operatorname{ker} \delta_{0,},(U)=0$, and $\delta_{0,},(U)$ is injective for $j=1$, $2, \cdots, n$. Therefore by Theorem $2.5, \delta_{0}, \mathrm{j}$ is injective for those values of $j$.

(b) This directly follows from the definition of $\delta_{-1}$, given preceding to the statement of Theorem 2.4 .

Now, Tensor our double complex $\left(C_{i, j}, \delta_{i, j}, d_{i, j}\right)$ with $R$-module $k$ to get a new double complex $\left(C_{i, j} \otimes_{R} k, \delta_{i, j} \otimes_{R} i d, d_{i, j} \otimes_{R} i d\right)$. Then clearly all the new vertical maps $d_{i, j} \otimes_{R} i d$ become zero, as $d_{i}, j$ are maps in Koszul complex resolutions (see [4]). Let $\theta_{i, j}=\delta_{i, j} \otimes_{R} i d$. 
Then the new tensored double complex looks like the following:

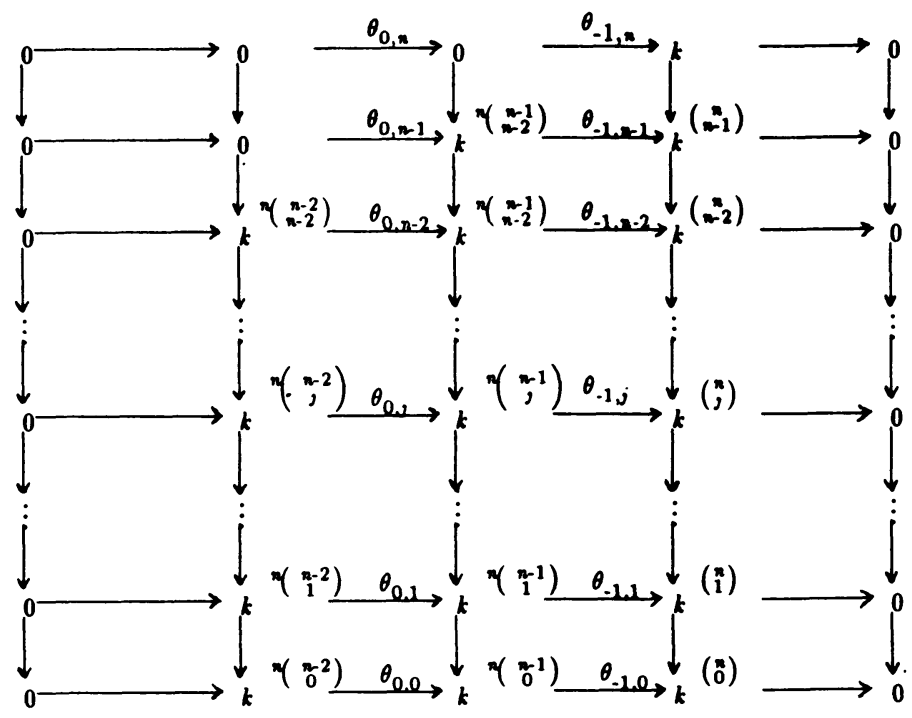

\section{THEOREM 2.9.}

(a) $\boldsymbol{\theta}_{0}, \mathrm{j}$ is injective for $j=1,2, \cdots, n$

(b) $\theta_{-1,}$, is surjective for $j=0,1, \cdots, n-1$.

PROOF. This directly follows from Theorem 2.8, since all the entries of matrices of the maps of $\delta_{0,}$, and $\delta_{-1}$, belong to the base field $k$.

The following theorem gives more information about the modules $H_{1}\left(C_{.,}, \delta_{.,}\right)$

THEOREM 2.10. $H_{0}\left(C_{.,}, \delta_{. j}\right), H_{-1}\left(C_{.,}, \delta_{. \jmath}\right)$ and $H_{-2}\left(C_{.,}, \delta_{. \jmath}\right)$ are free $R$-modules for $j=0,1, \cdots, n$ and we have

$$
\begin{aligned}
& \operatorname{rank}_{R}\left[H_{0}\left(C_{.,}, \delta_{. j}\right)\right]=\left\{\begin{array}{lll}
1 & j=0 \\
0 & j \neq 0
\end{array}\right. \\
& \operatorname{rank}_{R}\left[H_{-1}\left(C_{. j}, \delta_{. j}\right)\right]= \begin{cases}\left(\begin{array}{l}
n \\
j
\end{array}\right) \frac{(j-1)(n-j-1)}{n-1} & j=1,2, \cdots, n-1 \\
0 & j=0, n\end{cases} \\
& \operatorname{rank}_{R}\left[H_{-2}\left(C_{.,}, \delta_{. j}\right)\right]= \begin{cases}0 & j \neq n \\
1 & j=n\end{cases}
\end{aligned}
$$

Identical assertions hold for $\operatorname{dim}_{k}\left[H_{i}\left(C_{.,} \otimes_{R} k, \delta_{. j} \otimes_{R} i d\right)\right]$ for $i=0,-1,-2$.

PROOF. Most of the above assertions are easy consequences of Theorem 2.8 and Theorem 2.9, except perhaps the one for $\operatorname{rank}_{R}\left[H_{-1}\left(C_{. j}, \delta_{. j}\right)\right], j=1,2, \cdots, n-1$. But, it also follows without difficulty since,

$$
\begin{aligned}
\operatorname{rank}_{R}\left[H_{-1}\left(C_{.,}, \delta_{. \jmath}\right)\right] & =-\operatorname{rank}_{R} C_{0, j}+\operatorname{rank}_{R} C_{-1, j}-\operatorname{rank}_{R} C_{-2,} \\
& =-n\left(\begin{array}{c}
n-2 \\
j
\end{array}\right)+n\left(\begin{array}{c}
n-1 \\
j
\end{array}\right)-\left(\begin{array}{c}
n \\
j
\end{array}\right) \\
& =\left(\begin{array}{c}
n \\
j
\end{array}\right) \frac{(j-1)(n-j-1)}{n-1}
\end{aligned}
$$


Now, an important theorem of this paper.

THEOREM 2.11. Let $n \geq 3$. Then the $t^{t h}$ Betti number $\beta_{\imath}(n)$ of the $n$-gon is given by,

$$
\beta_{\imath}(n)=\left\{\begin{array}{cl}
1 & \imath=0 \\
\left(\begin{array}{c}
n \\
\imath+1
\end{array}\right) \frac{\imath(n-\imath-2)}{n-1} & i=n-2 \\
1 & \text { otherwise }
\end{array}\right.
$$
PROOF. This follows from Theorem 2.10 and the equation, $\operatorname{dim}_{k}\left[\operatorname{Tor}_{\imath}^{R}(R / I, k)\right]=\stackrel{n}{\stackrel{\oplus}{=}} 0$
$\operatorname{dim}_{k}\left[H_{\imath-}\left(C_{. j} \otimes{ }_{R} k, \delta_{. j} \otimes i d\right)\right]$ (See also Corollary 1.4 and Proposition 1.5 on page 7 of $[6]$ ).

EXAMPLE 2.11. For $n=5$, one obtains the pentagon. In this case,

$$
\begin{aligned}
& \Delta=\{\emptyset,\{1\},\{2\},\{3\},\{4\},\{5\},\{1,2\},\{2,3\},\{3,4\}\{4,5\},\{1,5\} \\
& R=k\left[x_{1}, x_{2}, x_{3}, x_{4}, x_{5}\right] \\
& I=\left(x_{1} x_{3}, x_{1} x_{4}, x_{2} x_{4}, x_{2} x_{5}, x_{3} x_{5}\right)
\end{aligned}
$$

Then using formula (2.2), the Betti numbers $\beta_{1}(5)$ of the pentagon are given by, $\beta_{0}(5)=1$, $\beta_{1}(5)=5, \beta_{2}(5)=5$, and $\beta_{3}(5)=1$.

\section{PASCAL TYPE PROPERTIES OF BETTI NUMBERS OF THE $\boldsymbol{n}$-GON.}

As done in the previous section, using formula (2.2), one can find the Betti numbers $\beta_{\imath}(n)$ of various $n$-gons, $n=3,4,5, \cdots$ and form the following array (3.1). It is known as the Triangle of Betti numbers. In that, if you consider any row, the 0th Betti number appears to the extreme left, the 1st Betti number appears to the right of that, $\cdots$, etc., etc.

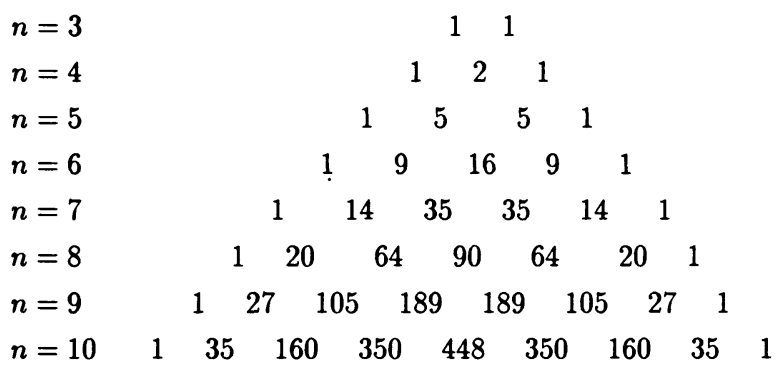

Notice that the above array consists of diamonds of the form,

$$
\beta_{i(n+1)} \beta_{i}(n)>\beta_{i+1}(n+2) \overbrace{i+1}^{(n+1)}
$$

Some interesting connections exist between the Betti numbers $\beta_{2}(n), \beta_{2}(n+1)$ and $\beta_{1+1}(n+1)$ at the top three vertices of the diamond, and the Betti number $\beta_{1+1}(n+2)$ at the bottom most vertex of the diamond. These properties are quite similar to the ones enjoyed by the Pascal's Triangle, concerning the binomial coefficients. Before proving them, we need two lemmas.

LEMMA 3.1. Let $n>7$ be a natural number. Then for $3<i \leq n-4$, we have, $n>\frac{i^{2}+2 i-1}{i-1}$.

PROOF. $i>3 \Rightarrow i^{2}+3 i-4>i^{2}+2 i-1 \Rightarrow(i+4)(i-1)>i^{2}+2 i-1 \Rightarrow i+4>\frac{i^{2}+2 i-1}{i-1} \Rightarrow$ $n \geq i+4>\frac{i^{2}+2 i-1}{i-1} \Rightarrow n>\frac{i^{2}+2 i-1}{i-1} \quad$ Q.E.D.

LEMMA 3.2. (The symmetry of the Betti numbers). For any positive integer $n \geq 3$ and $i=0,1, \cdots, n-2, \beta_{\mathbf{\imath}}(n)=\beta_{n-1-2}(n)$. 
PROOF. Let $n \geq 3$ be any positive integer. For $i=0$ or $n-2$, we clearly have the indicated result, by formula (2.2) for the Betti numbers $\beta_{\imath}(n)$. Therefore, let $i=1,2, \cdots, n-3$, which implies $n-\imath-2$ also takes values $1,2, \cdots, n-3$. Hence, again by formula (2.2),

$$
\begin{aligned}
\beta_{n-1-2}(n) & =\left(\begin{array}{c}
n \\
n-i-1
\end{array}\right) \frac{(n-i-2)(n-n+\imath+2-2)}{n-1}=\left(\begin{array}{c}
n \\
n-i-1
\end{array}\right) \frac{i(n-\imath-2)}{n-1} \\
& =\left(\begin{array}{c}
n \\
n+1
\end{array}\right) \frac{i(n-i-2)}{n-1}=\beta_{\imath}(n)
\end{aligned}
$$

THEOREM 3.3. (Pascal Type properties of Betti numbers).

(i) Let $n=3,4,5$ or 6 . Then for any $i=0,1,2, \cdots, n-2$ we have,

$$
\beta_{\imath}(n)+\beta_{\imath}(n+1)+\beta_{t+1}(n+1)<\beta_{t+1}(n+2)
$$

(ii) Let $n=7$. Then for $i=0,1,4$ and 5 we have,

$$
\beta_{1}(n)+\beta_{1}(n+1)+\beta_{1+1}(n+1)<\beta_{1+1}(n+2)
$$

and for $i=2,3$ we have,

$$
\beta_{i}(n)+\beta_{i}(n+1)+\beta_{i+1}(n+1)=\beta_{i+1}(n+2)
$$

(iii) Let $n>7$ be any positive integer. Then for $i=0,1, n-3, n-2$ we have,

$$
\beta_{\imath}(n)+\beta_{\imath}(n+1)+\beta_{\mathfrak{t}+1}(n+1)<\beta_{\mathfrak{1}+1}(n+2)
$$

and for $2 \leq i \leq n-4$ we have,

$$
\beta_{\mathfrak{i}}(n)+\beta_{\mathfrak{t}}(n+1)+\beta_{1+1}(n+1)>\beta_{1+1}(n+2)
$$

(iv) Let $n \geq 3$ be any positive integer. Then for any $i=0,1, n-3, n-2$ we have,

$$
\beta_{i}(n)+\beta_{i}(n+1)+\beta_{i+1}(n+1)<\beta_{i+1}(n+2)
$$

PROOF. (i) and (ii) follow at once from the triangle of Betti numbers (3.1) given at the beginning of this section.

Before proving (iii), we will obtain an expression for $\beta_{i}(n)+\beta_{\mathbf{l}}(n+1)+\beta_{i+1}(n+1)$ $-\beta_{\mathfrak{i}+1}(n+2)$ for $n>3$ and $1 \leq i \leq n-3$. Using the formula (2.2) for the Betti numbers $\beta_{\mathfrak{z}}(n)$, one obtains,

$$
\begin{aligned}
\beta_{i}(n)+ & \beta_{i}(n+1)+\beta_{i+1}(n+1)-\beta_{i+1}(n+2) \\
= & \left(\begin{array}{c}
n \\
i+1
\end{array}\right) \frac{i(n-i-2)}{n-1}+\left(\begin{array}{c}
n+1 \\
i+1
\end{array}\right) \frac{i(n-i-1)}{n} \\
& \quad+\left(\begin{array}{c}
n+1 \\
i+2
\end{array}\right) \frac{(i+1)(n-i-2)}{n}-\left(\begin{array}{c}
n+2 \\
i+2
\end{array}\right) \frac{(i+1)(n-i-1)}{n+1} \\
= & \frac{n !}{(i+1) !(n-i-1) !}\left\{\frac{(n-i-2)}{n-1}+\frac{i(n-i-1)}{(n-i) n}\right. \\
& \left.\quad+\frac{(n+1)(i+1)(n-i-2)}{(i+2) n}-\frac{(n+2)(i+1)(n-i-1)}{(i+2)(n-i)}\right\} \\
= & \frac{n !}{(i+1) !(n-i-1) !} \frac{\left[n(i-1)-i^{2}-2 i+1\right]}{n} \\
= & \left(\begin{array}{c}
n \\
i+1
\end{array}\right) \frac{\left[n(i-1)-i^{2}-2 i+1\right]}{n}
\end{aligned}
$$


Hence, for $n>3$ and $1 \leq i \leq n-3$ we have,

$$
\begin{aligned}
\beta_{i}(n)+\beta_{i}(n+1)+\beta_{\mathbf{t}}(n+1)-\beta_{\mathbf{i}+1}(n+2) \\
=\left(\begin{array}{c}
n \\
i+1
\end{array}\right) \frac{\left[n(i-1)-\imath^{2}-2 i+1\right]}{n}
\end{aligned}
$$

Now, to prove the second part of (iii), let $n>7$ be any positive integer, and $\imath$ be a positive integer such that $2 \leq i \leq n-4$. If $3<i \leq n-4$, using the Lemma 3.1 and the formula (3.2), we immediately obtain $\beta_{2}(n)+\beta_{1}(n+1)+\beta_{1+1}(n+1)-\beta_{1+1}(n+2)>0$. Since this result is true for $i=n-4$ and $i=n-5$, it is also true for $i=2$ and $i=3$ respectively, using Lemma 3.2 . Therefore, for $n>7$ and $2 \leq i \leq n-4$, the inequality $\beta_{1}(n)+\beta_{1}(n+1)+\beta_{1+1}(n+1)$ $>\beta_{i+1}(n+2)$ holds.

Finally, to prove (iv) (the first part of (iii) is also included in this statement), let $n \geq 3$ be any positive integer. For $i=0, \beta_{1}(n)+\beta_{\imath}(n+1)+\beta_{\imath+1}(n+1)-\beta_{\imath+1}(n+2)=\beta_{0}(n)+\beta_{0}(n+1)$ $+\beta_{1}(n+1)-\beta_{1}(n+2)=1+1+\frac{1}{2}(n+1)(n-2)-\frac{1}{2}(n+2)(n-1)=2-n<0$. For $n=3$ and $i=1$, the required result is clear from the triangle of Betti numbers (3.1). So assume that $n>3$ and $i=1$. Then by (3.2) one obtains, $\beta_{1}(n)+\beta_{1}(n+1)+\beta_{2}(n+1)-\beta_{2}(n+2)=\left(\begin{array}{l}n \\ 2\end{array}\right) \frac{(-2)}{n}<0$. Hence, we get for $n \geq 3$ and $i=0,1$ that $\beta_{i}(n)+\beta_{i}(n+1)+\beta_{i+1}(n+1)<\beta_{i+1}(n+2)$. Clearly, it is also true for $i=n-2, n-3$ using the symmetry stated in Lemma 3.2.

Q.E.D.

REMARK. We can illustrate the content of the above theorem by means of the diagram (3.3). In that, the positions of the diamonds satisfying $\beta_{8}(n)+\beta_{3}(n+1)+\beta_{1+1}(n+1)$ $\left\langle\beta_{1+1}(n+2)\right.$ are indicated by $\diamond$. No matter how indefinitely you continue the triangle of Betti numbers, there are only two diamonds which satisfy the equality $\beta_{3}(n)+\beta_{\mathfrak{a}}(n+1)$ $+\beta_{\mathfrak{t}+1}(n+1)=\beta_{\mathfrak{i}+1}(n+2)$, namely,

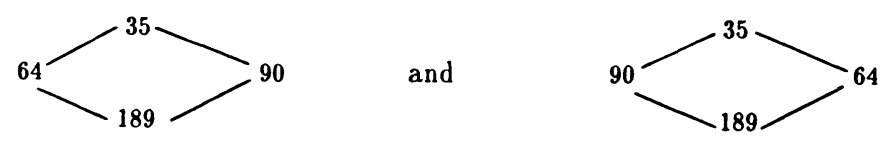

Except the above described ones, all other diamonds satisfy the inequality $\beta_{i}(n)+\beta_{i}(n+1)+\beta_{i+1}(n+1)>\beta_{i+1}(n+2)$.

$$
\begin{aligned}
& n=3 \\
& n=4 \\
& n=5 \\
& n=6 \\
& n=7 \\
& n=8 \\
& n=9 \\
& n=10
\end{aligned}
$$

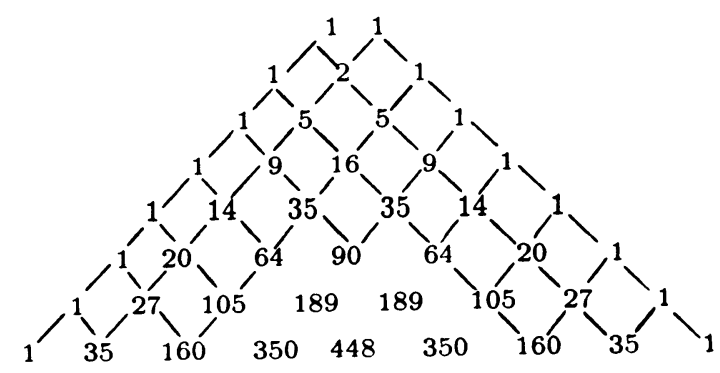

\section{REFERENCES}

1. HOCHSTER, M., Cohen-Macaulay rings, combinatorics and simplicial complexes, Ring Theory II, Proceedings of the Second Oklahoma Conference (1975), 171-223.

2. MUNKRES, J., Elements of Algebraic Topology, Addison-Wesley, 1984.

3. STANLEY, R., Combinatorics and Commutative Algebra, Birkhauser, 1983.

4. MATSUMURA, H., Commutative Ring Theory, Cambridge University Press, 1986.

5. DE ALWIS, T., Ph.D. Thesis, University of Minnesota, 1988.

6. JOHNSON, S., Ph.D. Thesis, University of Minnesota, 1982. 


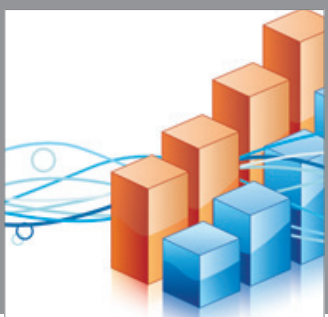

Advances in

Operations Research

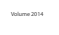

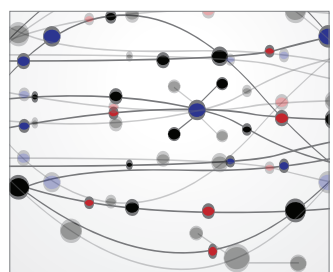

\section{The Scientific} World Journal
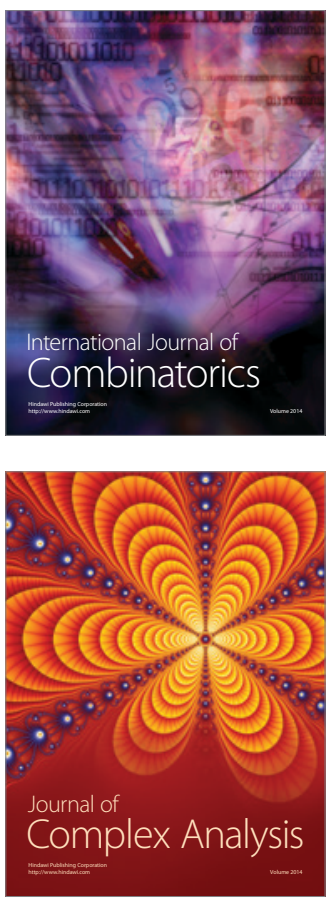

International Journal of

Mathematics and

Mathematical

Sciences
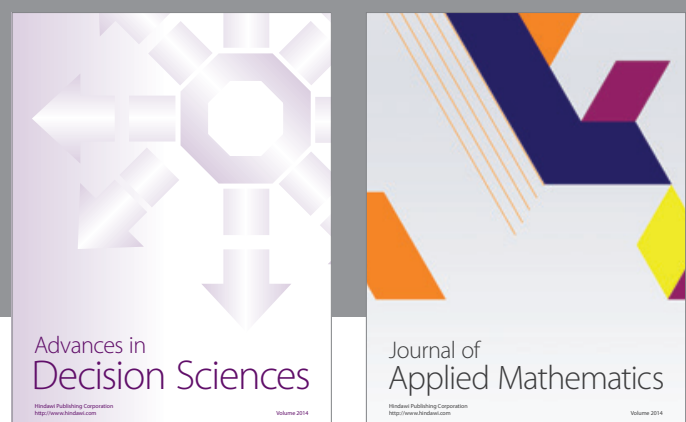

Journal of

Applied Mathematics
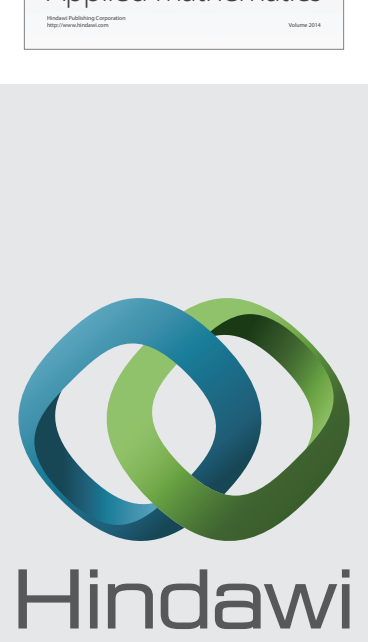

Submit your manuscripts at http://www.hindawi.com
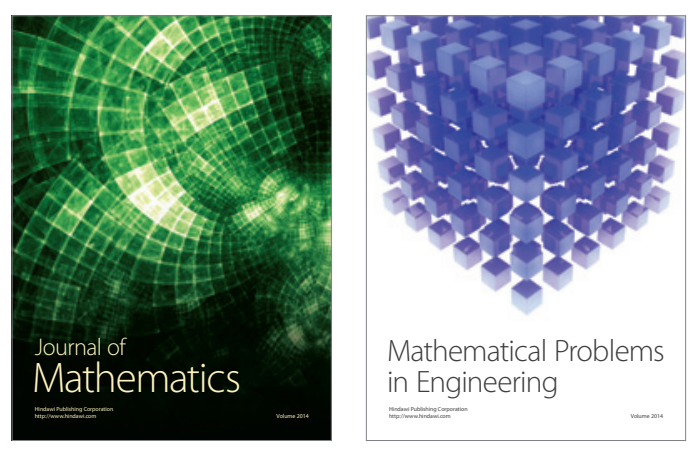

Mathematical Problems in Engineering
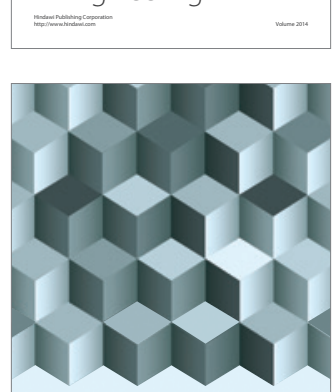

Journal of

Function Spaces
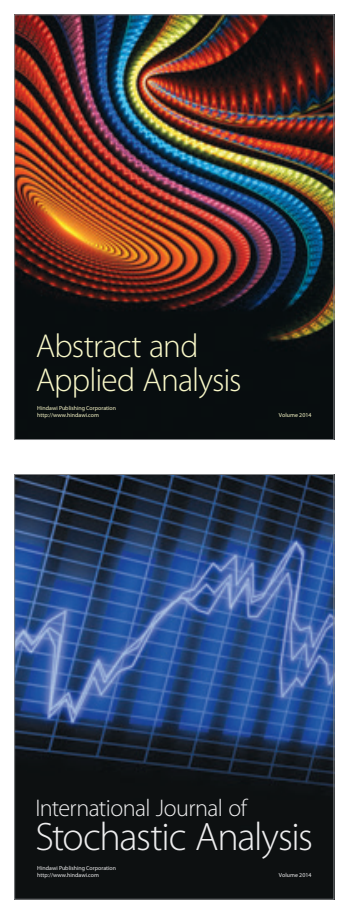

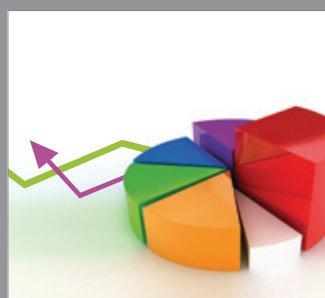

ournal of

Probability and Statistics

Promensencen
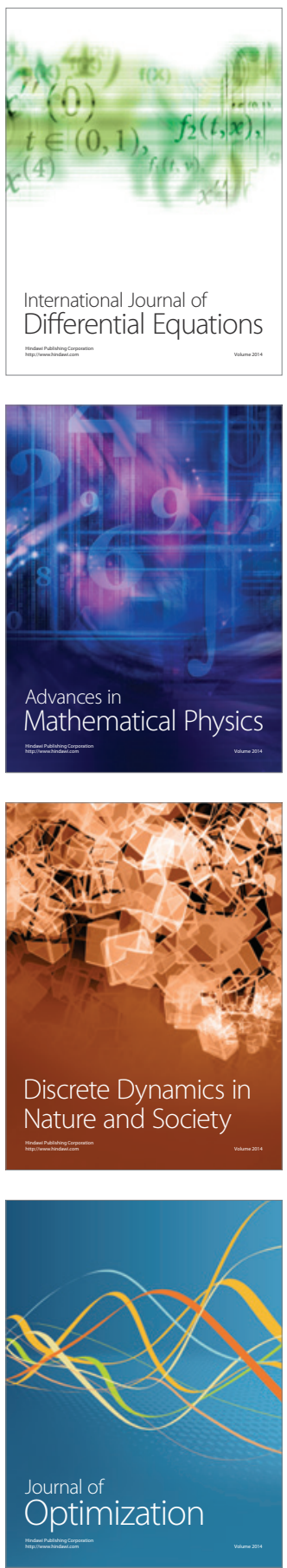